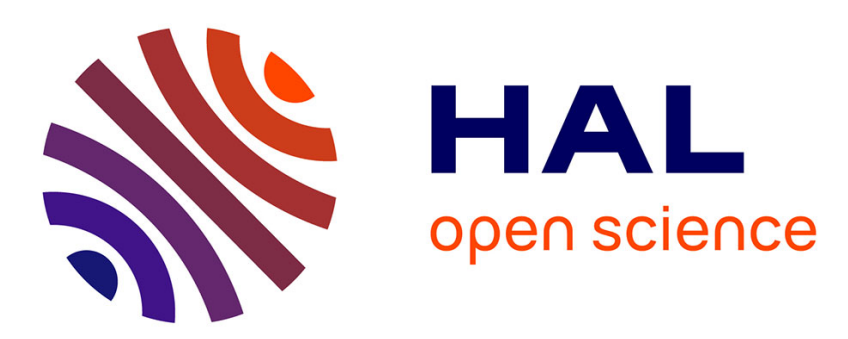

\title{
A safe and compact flow platform for the neutralization of a mustard gas simulant with air and light
}

Noémie Emmanuel, Pauline Bianchi, Julien Legros, Jean-Christophe Monbaliu

\section{To cite this version:}

Noémie Emmanuel, Pauline Bianchi, Julien Legros, Jean-Christophe Monbaliu. A safe and compact flow platform for the neutralization of a mustard gas simulant with air and light. Green Chemistry, 2020, 22 (13), pp.4105-4115. 10.1039/d0gc01142h . hal-02996914

\section{HAL Id: hal-02996914 https://hal.science/hal-02996914}

Submitted on 11 Nov 2020

HAL is a multi-disciplinary open access archive for the deposit and dissemination of scientific research documents, whether they are published or not. The documents may come from teaching and research institutions in France or abroad, or from public or private research centers.
L'archive ouverte pluridisciplinaire HAL, est destinée au dépôt et à la diffusion de documents scientifiques de niveau recherche, publiés ou non, émanant des établissements d'enseignement et de recherche français ou étrangers, des laboratoires publics ou privés. 


\title{
Green Chemistry
}

$<$ Received 00th January 20xx Accepted 00th January 20xx

DOI: $10.1039 / x 0 x x 00000 x$

\section{www.rsc.org/}

\section{A safe, green and compact flow platform for the neutralization of a mustard gas simulant with air and light}

\author{
Noémie Emmanuel, ${ }^{\mathrm{a}, \mathrm{Il}}$ Pauline Bianchi, ${ }^{\mathrm{a}, \mathrm{Il}}$ Julien Legros ${ }^{\mathrm{b}}$ and Jean-Christophe M. Monbaliu ${ }^{\mathrm{a}, *}$
}

A low footprint, mobile, robust and frugal chemical neutralization technology is reported for the oxidative neutralization of a mustard gas simulant. It relies on the inherent properties of a highly engineered continuous flow setup and carefully optimized and simple, yet robust, experimental conditions. The neutralization protocol uses only non-toxic, widely available and cheap chemicals. The continuous flow setup integrates a singlet oxygen generator and exploits its oxidative power to neutralize 2-chloroethyl ethyl sulfide (CEES), the most common thioether mustard gas simulant. The flow reactor can be connected to either pressurized oxygen or air and handles CEES as a $1 \mathrm{M}$ solution in EtOH containing a trace amount $(0.06 \mathrm{~mol} \%)$ of a non-toxic and widely available photosensitizer (Methylene Blue). Upon irradiation with visible light (orange or white light), total and highly selective neutralization towards the corresponding non-toxic sulfoxide (1chloro-2-(ethylsulfinyl)ethane, CEESO) is obtained with reactor effluents containing less than $1 \%$ of the corresponding potentially toxic sulfone (1-chloro-2-(ethylsulfonyl)ethane, $\mathrm{CEESO}_{2}$ ). With a low footprint ( $\left.\mathrm{L} \times \mathrm{W} \times \mathrm{H} 94 \times 42 \times 40 \mathrm{~cm}\right)$, this neutralization technology can be embarked on a vehicle for on-site interventions, localized at a neutralization facility or both. The experimental work is also supported with a computational rationalization of the reactivity of CEES towards singlet oxygen.

\section{Introduction}

Chemical warfare agents (CWAs, Figure 1) were conceived in the darkest converging alleys of Science and Industry to optimize debilitating, incapacitant or lethal activity for maintaining military superiority. ${ }^{1}$ World War II stressed the emergence of much more redoubtable nuclear warfare, even though large stockpiles of CWAs were maintained worldwide and new chemical agents were developed. Since WWII, CWAs were sporadically used for armed conflicts with the last reports dating back to 2017-2018 in the Middle East. The tragedy with CWAs remains that it also affects civilians who lack protection gears and antidotes. ${ }^{2}$

The Chemical Weapons Convention (CWC) entered in force in 1997 with the ambition to prohibit the possession, manufacture and use of CWAs and was ratified by 193 countries. $^{3}$ Besides prohibition, it also aims at developing resources, procedures and capacities to treat the

a. Center for Integrated Technology and Organic Synthesis, MolSys Research Unit, University of Liège, B-4000 Liège (Sart Tilman), Belgium

jc.monbaliu@uliege.be www.citos.uliege.be

${ }^{b .}$ Normandie Université, INSA Rouen, UNIROUEN, CNRS COBRA laboratory (UMR 6014 \& FR3038) 76000 Rouen (France)

"Equal contributions.

Electronic Supplementary Information (ESI) available: fluidic components, experimental procedures, details of the fluidic setup and procedures, computational details, details for the off-line analyses (GC, HPLC) and structural assignments (NMR). See DOI: 10.1039/x0xx00000x consequences of a CWA attack and to supervise the destruction of stockpiles, related equipment and infrastructure. ${ }^{2,4}$

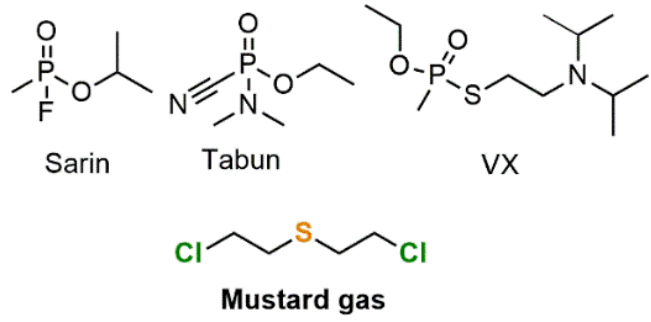

Fig. 1. Representative examples of Schedule I CWAs.

There are still large inventories of CWAs reminiscent of previous conflicts, either as a latent, still usable threat or as spoiled ammunitions. Besides, the availability of various chemicals on small to medium scale as well as emerging process technologies with a very low footprint have also raised significant concerns with the potential local production of CWAs for chemical terrorism. ${ }^{5}$ Following the inception of the CWC, a total of 8 countries declared CWAs, 7 of which have now completed the destruction of $97 \%$ of the declared inventories worldwide. The USA has announced the destruction of $90 \%$ of its total inventory and aims at a complete destruction by $2023 .{ }^{6,7}$ 
Among CWAs, 1-chloro-2-[(2-chloroethyl)sulfanyl]ethane (CAS 505-60-2) commonly referred to as mustard gas or HD is a Schedule I CWA with cytotoxic and vesicant properties. ${ }^{8}$ HD is considered as the parent molecule of sulfur mustards. The unique reactivity and toxicity profile of sulfur mustards arise from the combination of donor lone pairs $n_{\mathrm{s}}$ on the sulfur atom in the vicinity of two $\sigma^{*}{ }_{c-c l}$ acceptors, hence potentially forming strongly alkylating and toxic episulfonium species epiHD (Figure 2a). ${ }^{9}$ The most common method of destruction of HD relies on incineration, ${ }^{10,11}$ although such a method raises significant safety concerns not only for the emission of toxic gaseous or liquid emissions upon thermal treatment but also for the transportation risk. ${ }^{12}$ Chemical neutralization methods ${ }^{10,11}$ (methods 1-3, Figure 2b) for sulfur mustards such as HD or its most common simulant CEES (2-chloroethyl ethyl sulfide) typically target either one of the lone pairs $n_{\mathrm{S}}$ on sulfur through selective oxidation towards a sulfoxide (method 1 ) or the $\sigma^{*}{ }_{\mathrm{c}-\mathrm{Cl}}$ acceptors through hydrolysis or displacement with a strong nucleophile (method 2). ${ }^{13} \mathrm{~A}$ third alternative method of chemical neutralization relies on dehydrohalogenation protocols (method $\mathbf{3}$ ).

a.

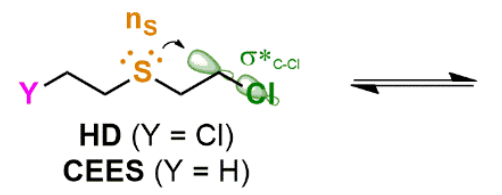

b.

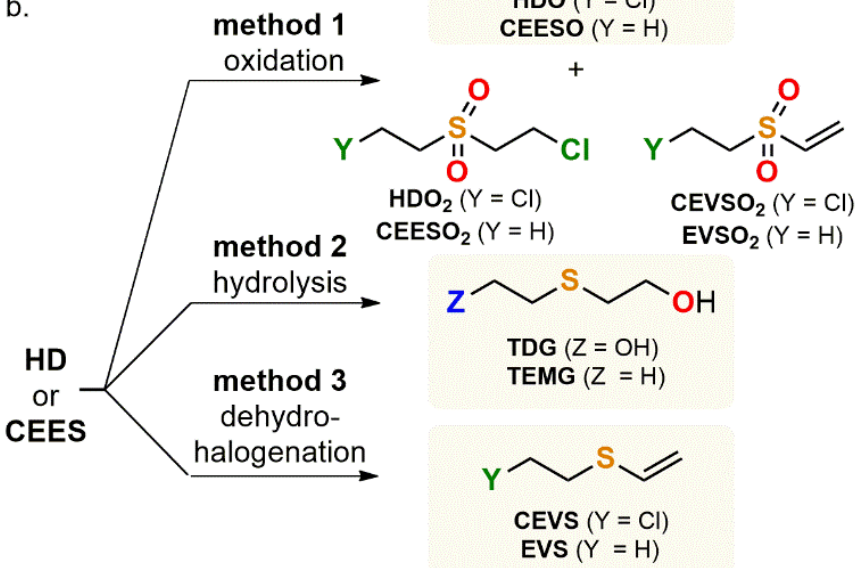

Method 1. Oxidation; Method 2. Hydrolysis; Method 3. Dehydrohalogenation. Compounds with the light gray background are considered low-toxicity neutralized species.

Although these oxidative neutralization methods seem straightforward, the complex reaction profile of HD/CEES makes them very cumbersome. For instance, the overoxidation of HD/CEES leads to the formation of a strong Michael acceptor sulfone $\mathrm{CEVSO}_{2} / \mathrm{EVSO}_{2},{ }^{14-16}$ the toxicity of which is comparable to the parent mustard. In all instances, its formation must thus be limited to trace amounts. The selectivity of the oxidation is therefore a critical parameter for the validation of a neutralization method. The lipophilic nature of HD/CEES typically renders aqueous oxidation protocols inefficient, hence often imposing surfactants ${ }^{14}$ or organic solvents as carriers. ${ }^{15}$ Primary oxidizers typically include hydrogen peroxide, ${ }^{14,16-22}$ peracids $^{15}$ or oxygen ${ }^{22-25}$ in the presence of homogeneous ${ }^{26}$ or heterogeneous catalysts. ${ }^{16-}$ 18,27-31 Other oxidizers were also reported such as $\mathrm{N}$ iodosuccinimide/water (Table 1 ). ${ }^{32}$

The oxidative neutralization methods relying on reactive oxygen species are very attractive, especially when molecular oxygen is the primary oxidant source (Table 1). Zeolites ${ }^{2}$ and MOF-based heterogeneous materials and fibers were also reported and successfully tested for the UV photooxidation of CEES and actual samples of HD..$^{23,28,33-36}$ These photooxidations typically proceed with complete conversion and high to complete selectivity towards the sulfoxide HDO with a catalytic amount (typ. 1-2 mol\%). ${ }^{23,28}$ The heterogeneous MOF photocatalyst can be easily recovered after neutralization and is potentially reusable. ${ }^{35}$ Photoactive ferrocene-conjugated microporous polymers were also reported. ${ }^{24,25}$ Despite excellent results, these methods have only been reported on the milligram scale and would face additional challenges for reaching larger scales.

Organic homogeneous photosensitizers were also considered under aerobic conditions such as Riboflavin tetraacetate (RFTA) or riboflavin (vitamin B2). ${ }^{37,38}$ Under irradiation with blue LEDs $(450 \mathrm{~nm}$ ) with 2 mol\% RFTA at room temperature, CEES was quantitatively and selectively converted into CEESO in $10 \mathrm{~min}$ of irradiation. Borondipyrromethene (BODIPY) PSs were assessed for the photooxidation of CEES and actual samples of HD under blue light irradiation in solution or impregnated on PVDF films and textiles, providing CEES half-life down to $0.8 \mathrm{~min}^{39}$

Fig. 2. a. Reactivity and toxicity of HD; b. General chemical neutralization methods for mustard gas (HD) and its most common simulant (CEES). 
Table 1. Selection of procedures for the neutralization of mustard gas HD and its simulant CEES

\begin{tabular}{|c|c|c|c|c|c|c|c|}
\hline Entry & Author & Scale & Type & Catalyst & Oxidant & Solvent & Conditions \\
\hline 1 & Jackowski $^{57}$ & $5 \mathrm{mM}$ & Batch $^{a}$ & Rhodococcus R. IGTS8 & N.A. & $\mathrm{H}_{2} \mathrm{O}$ & $30^{\circ} \mathrm{C}, 14 \mathrm{~d}$, quant. ${ }^{\mathrm{c}}$ \\
\hline 2 & Landry $^{29}$ & $5.4 \mathrm{mg}$ & $\begin{array}{c}\text { Batch } \\
\mathrm{b}\end{array}$ & V-mesoporous $\mathrm{SiO}_{2}, 10 \%$ & $t-\mathrm{BuOOH}$ & $\mathrm{CH}_{2} \mathrm{Cl}_{2}$ & r.t., $1 \mathrm{~h}, 97 \%^{\mathrm{d}}$ \\
\hline 3 & Wynne $^{30}$ & $0.85 \mathrm{mg}$ & $\begin{array}{c}\text { Batch } \\
\mathrm{b}\end{array}$ & ZnOPPc & Air & neat & $\lambda, 32-34{ }^{\circ} \mathrm{C}, 24 \mathrm{~h}$, quant. ${ }^{\mathrm{e}}$ \\
\hline 4 & Cibulka $^{38}$ & $25 \mathrm{mg}$ & Batch $^{a}$ & riboflavin tetraacetate, 2 mol\% & $\mathrm{O}_{2}$ & $\mathrm{MeCN} / \mathrm{H}_{2} \mathrm{O}$ & $450 \mathrm{~nm}$, r.t. $10 \mathrm{~min}$; quant. \\
\hline 5 & Farha $^{28}$ & $25 \mathrm{mg}$ & $\begin{array}{c}\text { Batch } \\
\mathrm{b}\end{array}$ & NU-1000, 1 mol\% & $\mathrm{O}_{2}$ & $\mathrm{MeOH}$ & $\sim 400 \mathrm{~nm}$, r.t., 15 min, quant. ${ }^{c}$ \\
\hline 6 & Legros $^{20}$ & $\begin{array}{c}\sim 780 \\
\text { g.day }{ }^{-1}\end{array}$ & Flow $^{a}$ & \multicolumn{2}{|c|}{ methanesulfonic acid urea- $\mathrm{H}_{2} \mathrm{O}_{2}$ (UHP) adduct } & $\mathrm{MeOH}$ & r.t., 3.9 min, quant. ${ }^{c}$ \\
\hline 7 & Farha $^{34}$ & $25 \mathrm{mg}$ & $\begin{array}{c}\text { Batch } \\
\mathrm{b}\end{array}$ & NU-1000-PCBA, 0.7 mol\% & $\mathrm{O}_{2}$ & $\mathrm{MeOH}$ & $400 \mathrm{~nm}, 14 \mathrm{~min}$, quant. ${ }^{c}$ \\
\hline 8 & Clarck $^{26}$ & $28.7 \mathrm{mg}$ & $\begin{array}{c}\text { Batch } \\
\text { b }\end{array}$ & $\mathrm{Cu}(\mathrm{hfac})_{2} \cdot \mathrm{H} 2 \mathrm{O}, 4 \mathrm{~mol} \%$ & $t-\mathrm{BuOOH}$ & $\mathrm{CDCl}_{3}$ & r.t., $27 \mathrm{~h}, 50 \%^{\mathrm{c}}$ \\
\hline 9 & Zhao $^{22}$ & $10 \mathrm{mg}$ & Batch $^{a}$ & / & $\mathrm{H}_{2} \mathrm{O}_{2}$ & {$[\mathrm{BMIm}] \mathrm{HCO}_{3}$} & r.t., 30 min, quant. ${ }^{f}$ \\
\hline 10 & Karwacki ${ }^{39}$ & $\begin{array}{c}172 \text { mM } \\
\text { no info }\end{array}$ & Batch $^{a}$ & BODIPYs & $\mathrm{O}_{2}$ & $d 4-\mathrm{MeOH}$ & $3-30$ min, quant. ${ }^{\mathrm{g}}$ \\
\hline 11 & Karwacki $^{39}$ & $\begin{array}{c}2.8 \mathrm{mg} \\
\text { (HD) }\end{array}$ & $\begin{array}{c}\text { Batch } \\
\mathrm{b}\end{array}$ & BODIPY-I in fabric, 0.1 mol\% & Air & neat & r.t. 30 min, quant. ${ }^{g}$ \\
\hline 12 & Farha $^{33}$ & $25 \mathrm{mg}$ & $\begin{array}{c}\text { Batch } \\
\mathrm{b}\end{array}$ & PCN-222, UMCM-313, 1 mol\% & $\mathrm{O}_{2}$ & $\mathrm{MeOH}$ & $395-420 \mathrm{~nm} 12-18 \mathrm{~min}$, quant. ${ }^{\mathrm{c}}$ \\
\hline 13 & Farha $^{35}$ & $25 \mathrm{mg}$ & $\begin{array}{c}\text { Batch } \\
\mathrm{b}\end{array}$ & {$\left[\mathrm{Y}(\mathrm{H} 9 \mathrm{TPPA})\left(\mathrm{H}_{2} \mathrm{O}\right)_{\mathrm{x}}\right] \mathrm{Cl}_{2} \cdot \mathrm{yH}_{2} \mathrm{O}$} & $\mathrm{O}_{2}$ & $\mathrm{MeOH}$ & $400-500 \mathrm{~nm}, 28 \mathrm{~min}$, quant. ${ }^{\mathrm{c}}$ \\
\hline 14 & Zafrani $^{13}$ & $50 \mathrm{mg}$ & $\begin{array}{c}\text { Batch } \\
\mathrm{b}\end{array}$ & Me-DABCOF, 6-20 wt\% & N.A. & $\mathrm{H}_{2} \mathrm{O}$ & 36 min -24 h, quant. ${ }^{c}$ \\
\hline 15 & Estour $^{21}$ & $116,5 \mathrm{mg}$ & $\begin{array}{c}\text { Batch } \\
\mathrm{b}\end{array}$ & MIP/porphyrin, 0.25 mol\% & $\mathrm{H}_{2} \mathrm{O}_{2}$ & $\mathrm{MeOH} / \mathrm{H}_{2} \mathrm{O}$ & r.t., 2 h, $99.3 \%^{c}$ \\
\hline 16 & This work & $\begin{array}{l}180 \mathrm{~g} \\
\text { day }^{-1}\end{array}$ & Flow $^{a}$ & Methylene Blue, 0.06 mol\% & $\mathrm{O}_{2}$ or air & EtOH & $610 \mathrm{~nm}, 4 \mathrm{~min},>99 \%{ }^{\mathrm{h}}$ \\
\hline
\end{tabular}

${ }^{a}$ Homogeneous conditions; ${ }^{b}$ heterogeneous conditions; ${ }^{c}$ no sulfone detected; ${ }^{d}$ CEESO/CEESO 2 ratio of $3.1 ;{ }^{e}$ trace amounts for long reaction time; ${ }^{f} 6 \%$ of CEESO ${ }_{2}{ }^{\mathrm{g}}$ trace amounts; ${ }^{\mathrm{h}}<1 \%$ of CEESO2

Other critical parameters are the compactness, robustness and inherent safety related to the neutralization method. Indeed, the neutralization setup must be transportable and compact for rapid deployment on site. It must also provide consistent neutralization with high selectivity yet relying on simple, non-toxic, cheap and widely available chemicals. While most of the oxidative procedures rely on batch setups, several authors have considered continuous flow conditions and flow reactors as process technologies endowed with unique inherent features that could be exploited for the efficient oxidation of CEES specifically -for the use of oxygen and related reactive species. ${ }^{40-45}$ In particular, photocatalysis in flow has attracted significant attention both for the preparation of advanced chemical structures and for the chemical destruction (neutralization) of pollutants. ${ }^{46}$ Flow protocols relying on either homogeneous or heterogeneous photocatalysts ${ }^{47-52}$ benefit from the synergistic combination of flow and photochemistry: uniform irradiation with accurately controlled irradiations times, hence leading to intensified reactions with shorter and more selective reaction pathways. ${ }^{46,53-55}$ Flow conditions are also typically considered as scale-independent and easier to scale-up than conventional batch setups. ${ }^{46,53-56}$

Seeberger was actually one of first to report the catalytic photooxidation of thiodiglycol TDG $(0.25 \mathrm{M})$ under continuous flow conditions. ${ }^{58}$ The authors used tetraphenylporphyrin (TPP, $2 \mathrm{mM}$ ) in chloroform as a photosensitizer. The reactor was operated at room temperature under 6.9 bar of 
counterpressure and under UV irradiation (450 W medium pressure mercury lamp). Analog TDG was oxidized with 95\%, yielding to a 3:1 mixture of the corresponding sulfoxide and sulfone. Legros reported a compact flow process with an integrated in-line low field $\mathrm{NMR}^{20}$. The oxidation was carried out with the hydrogen peroxide/urea complex (UHP) in methanol with methanesulfonic acid as promotor. The authors reported complete and selective oxidation mustard gas simulant CEES within $3.9 \mathrm{~min}$ of residence time, and overoxidation to the sulfone was avoided by collecting the reaction effluents in a quenching solution. Despite its effectiveness, their procedure imposes the availability and transportation of explosive and corrosive reagents along with the reactor setup. Moreover, the use of UHP releases stoichiometric amounts of urea in the effluents to be further disposed.

Based upon our expertise in the design of flow processes and in particular for the generation and handling of reactive oxygen species ${ }^{5,59-61}$, a compact flow system $(\mathrm{L} \times \mathrm{W} \times \mathrm{H} 94 \times 42$ $x 40 \mathrm{~cm}$ ) was devised for enabling rapid deployment on site and effective oxidative neutralization with frugal and benign resources. We report herein an advanced continuous flow setup for the selective oxidation of simulant CEES and further document the reactivity of such thioethers through MP2 and Natural Bond Order (NBO) computations. The combination of both experimental and theoretical approaches led to a more refined understanding of the unique features of the photooxidation of thioethers. This process is unique compared to the prior Art (Table 1, entry 16) since (a) it relies on the unique features of a highly engineered continuous flow setup using intensified and user-friendly conditions; (b) it uses the powerful combination of oxygen (or air), visible light and trace amounts (0.06 mol\%) of a non-toxic, cheap and widely available organic photosensitizer (Methylene Blue); (c) only environmental and user-friendly reagents are involved, including the solvent (EtOH); (d) the reactor setup is mobile and can be embarked on a vehicle for on-site neutralization with minimal resources and it can be integrated with in-line analysis for process monitoring and increased safety; (e) quantitative conversion of simulant CEES is obtained with high selectivity within $4 \mathrm{~min}$ of irradiation time under mild process conditions $\left(20^{\circ} \mathrm{C}\right.$, 9 bar) with unprecedented concentrations ( 1 $\mathrm{M}$ in ethanol); ( $\mathrm{f}$ ) the process is virtually scaled-independent since it relies on intensified flow conditions that can be transposed to much larger scales with larger commercial fluidic reactors. It provides the most cost effective, operatorfriendly and low footprint process with high atom economy reported so far for the oxidative neutralization of mustard gas simulant CEES.

\section{Experimental section}

General information
Conversion, selectivity and yield were determined by Gas Chromatography coupled to Flame Ionization Detection (GCFID) or Mass Spectrometry (GC-MS) or by High Performance Liquid Chromatography coupled to Diode-Array Detection (HPLC-DAD). GC and HPLC conversions and yields were determined using external calibration curves established with commercial standards (2-chloroethylethyl sulfide, 2chloroethylethyl sulfone, ethyl vinyl sulfide, ethyl vinyl sulfone, methyl vinyl sulfone, methyl phenyl sulfide, methyl phenyl sulfoxide, methyl phenyl sulfone, dipropyl sulfide, dipropyl sulfone, thiodipropionic acid, dibenzyl sulfide, dibenzyl sulfoxide, dibenzyl sulfone, diphenyl sulfide, diphenyl sulfoxide, diphenyl sulfone, tetrahydrothiophene sulfide, tetrahydrothiophene sulfoxide, tetrahydrothiophene sulfone, benzyl phenyl sulfide, diethyl sulfide, benzyl methyl sulfide, benzyl methyl sulfone, dibenzothiophene, dibenzothiophene sulfone) or with synthesized reference samples following reported procedures (see Supporting Information Section 2.2.4 for experimental procedures). ${ }^{19,30,35,39}$ Structural identity was confirmed by ${ }^{1} \mathrm{H}$ and ${ }^{13} \mathrm{C}$ NMR spectroscopy (400 MHz Bruker Avance spectrometer) in $\mathrm{CDCl}_{3}$ (Supporting Information Sections 2.4 and 2.5). Ethanol, acetonitrile, water, Rose Bengal, Methylene Blue, 9,10-dicyanoanthracene, oxygen and air were obtained from commercial sources, and used without additional purification. CAUTION: 1-chloro-2(ethylsulfinyl)ethane (CEES) is a highly toxic and severe vesicant. It must be handled with great care under a fume hood. The access to 1-chloro-2-[(2-chloroethyl)sulfanyl]ethane (mustard gas or HD) is subjected to military clearance under very strict conditions.

\section{Computations}

Computations were performed at the $\mathrm{MP2} / 6-31+\mathrm{G} * *$ or B3LYP/6-31+G** level of theory with the Gaussian 09 package of programs (Revision D.01) with implicit solvent (PCM, $\varepsilon=$ 24.852, ethanol). Stationary points were optimized with gradient techniques (tight optimization convergence). Transition states were localized using the Newton-Raphson algorithm, and the nature of the stationary points was determined by analysis of the Hessian matrix. Intrinsic reaction coordinate (IRC) calculations were performed on representative transition states. NBO calculations and population analysis were performed at the B3LYP/6$31+G^{* *}$ level on the optimized structures (MP2/6-31+G**). Graphical representations of the HOMO of thioethers were obtained through the FormChk utility in Gaussian $09^{62}$.

\section{Experimental setup}

Mesofluidic setup. Mesofluidic experiments were carried with a Corning ${ }^{\circledR}$ Advanced-Flow $^{\mathrm{TM}}$ Lab Photo Reactor (1 fluidic module, $2.6 \mathrm{~mL}$ internal volume) and in a Corning ${ }^{\circledR}$ AdvancedFlow $^{\mathrm{TM}}$ LF/G1 skid Photo Reactor (5 fluidic modules integrated with static mixers and connected in series, $13 \mathrm{~mL}$ total internal volume). Feed and collection lines consisted of PFA tubing 
(1/8" o.d. or $1 / 4$ " o.d.) equipped with PFA or SS Swagelok connectors and ferrules. The liquid feed was handled with ThalesNano MicroHPLC pumps, and the gas feed (oxygen or air) was handled with a Bronkhorst EL FLOW Prestige mass flow controller. The reactor was maintained at reaction temperature with a LAUDA Integral XT 280 thermostat. The LED panels were maintained at $10{ }^{\circ} \mathrm{C}$ with a LAUDA RP845. Downstream pressure was regulated with a back pressure regulator from Zaiput Flow Technologies (BPR-10 or BPR-100). Optionally, an in-line IR (FlowIRTM from Mettler-Toledo equipped with a DTGS detector using HappGenzel apodization, a Silicon probe connected via a FlowIRTM sensor and a high pressure heated $10 \mu \mathrm{L}$ cell) or NMR (43 MHz Spinsolve ${ }^{\mathrm{TM}}$ carbon NMR spectrometer from Magritek $^{\circledR}$ ) was inserted downstream (Supporting Information Section 2.3).

\section{Typical run}

Continuous flow photochemical neutralization of CEES with oxygen. The pump used to deliver the solution of 2-chloroethyl ethyl sulfide (CEES, $1 \mathrm{M}$ ) and Methylene Blue (560 $\mu \mathrm{M})$ in EtOH was set to $1 \mathrm{~mL} \mathrm{~min}^{-1}$. The mass flow controller used to deliver oxygen was set to $20 \mathrm{~mL}_{\mathrm{N}} \mathrm{min}^{-1}$, and both streams were conveyed through PFA tubing to the first glass fluidic module of the Corning ${ }^{\circledR}$ Advanced-Flow $^{\mathrm{TM}}$ LF/G1 skid Photo Reactor upon irradiation at $610 \mathrm{~nm}$ (full intensity, 300 LEDs) at $20^{\circ} \mathrm{C}(9$ bar of counterpressure) (Figure 6). The reactor effluent was collected at steady state, diluted with $\mathrm{EtOH}$ and analyzed by GC/FID (99\% conversion, 99\% selectivity, see Table 1).

\section{Results and Discussion}

The photooxidation of thioethers has been widely discussed in the literature through mechanistic and computational studies, and various mechanisms were postulated and assessed. ${ }^{63-77}$ Depending on the photosensitization, the oxidation can proceed either through the addition of the thioether substrate onto singlet oxygen or through electron transfer (ET) involving the superoxide anion. ${ }^{66,78}$ The photooxidation of thioethers with singlet oxygen is consensually recognized as a complex reaction sequence leading to various oxidations products, among which the corresponding sulfoxides are typically the major product, along with variable amounts of sulfones and oxidative fragmentation products. ${ }^{65,69,74,76}$ The process goes through the formation of several key intermediates (Figure 3), the first of which being a weakly bound peroxysulfoxide intermediate $\mathbf{A}$ that forms upon the addition of the nucleophilic thioether substrate onto electrophilic singlet oxygen. Intermediate $\mathbf{A}$ was actually never isolated. The fate of the later determines the output of the photochemical process. Intermediate A typically undergoes physical quenching in apolar aprotic solvents, while it is stabilized in protic solvents, hence drastically improving the efficiency of the photooxidation. ${ }^{73,79}$ The exact nature of the second intermediate $\mathbf{B}$ has fed intense debate in the literature; advanced mechanistic and computations studies pointed towards hydroperoxysulfonium ylide $\mathbf{B}_{\mathbf{2}}$ rather than thiadioxirane $\mathbf{B}_{\mathbf{1}} \cdot{ }^{63-77}$ The reaction of each molecule of singlet oxygen can potentially result in 2 equiv. of sulfoxide. ${ }^{64,73}$

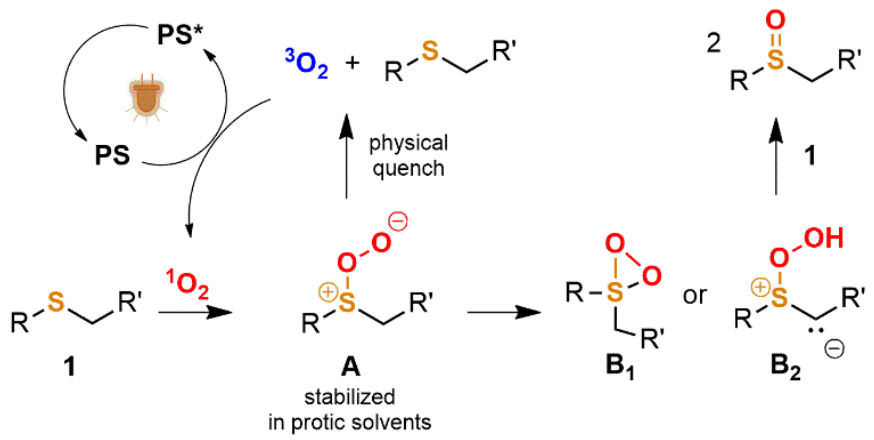

Fig. 3. Commonly accepted intermediates and mechanism for the reaction of thioethers with singlet oxygen under photosensitized conditions. Singlet oxygen is typically generated from ground state molecular oxygen upon irradiation in the presence of a photosensitizer (PS). A common organic PS is Methylene Blue.

The access to HD is subjected to military clearance under very strict conditions, while small inventories of its most widely accepted simulant (CEES) can be purchased. CEES is however still a very toxic and severe vesicant that must be handled with great care, hence precluding its direct use for large-scale optimization. Decision was made to undertake a preliminary computational study on the reactivity of HD and CEES towards singlet oxygen for comparison with other widely available non-toxic thioethers, the handling of which for preliminary experiments with singlet oxygen would not raise a significant safety hazard. Obviously, the model thioethers must echo the peculiar inherent reactivity of HD/CEES for enabling fast transposition with minimal readjustments to CEES. The rationale behind such an approach was to limit the exposure to extremely toxic chemicals upon the optimization of the process conditions. In silico modeling enables to compute quantitative or semi-quantitative models and data, which are particularly relevant when high toxicity chemicals are at stakes.

The efficiency of singlet oxygen oxidation of thioethers is not only very sensitive to reaction conditions, but it also critically depends on the inherent structural and stereoelectronic features of the thioether substrate. The availability of the non-bonding $n_{S}$ orbitals on the sulfur atom, which correlates to its intrinsic nucleophilicity, is thus paramount to ensure quick and efficient scavenging of singlet oxygen to form peroxysulfoxide intermediate A. Any factor that potentially decreases the availability of the non-bonding $n_{s}$ orbitals on the sulfur atom will therefore potentially reduce the efficiency of the reaction with singlet oxygen. As discussed in the Introduction (Figure 2a), the reactivity and toxicity profiles of sulfur mustards HD and CEES arise from the combination of a donor lone pair $n_{S}$ on the sulfur atom in the vicinity of two $\sigma^{*}{ }_{c-c l}$ acceptors; such stereoelectronic feature might thus impact the oxidative neutralization process. It is therefore critical to have access to a metric for assessing the extent of such stereoelectronic features for supporting the rational selection of an appropriate non-toxic model thioether. 
In order to quantify the availability of the non-bonding $n_{\mathrm{S}}$ orbitals and thus to provide insights on the potential for reaction with singlet oxygen, a preliminary computational study was undertaken on a series of model thioethers with defined structural and stereoelectronic features (Figure 4). The selected model thioethers included CEES and HD, as well as other alkyl, aryl and benzyl thioethers (1a-f),more specifically, dipropyl sulfide (1a), (chloromethyl)(ethyl)sulfide (1b), benzyl methyl sulfide (1c) methyl phenyl sulfide (1d), diphenyl sulfide (1e) and dibenzothiophene (1). Thus the selection of thioethers 1a-f included thioethers with qualitatively expected high (1a), intermediate (1c, 1d) and low (1b, 1e, 1f) availability of non-bonding $n_{S}$ orbitals, thus mirroring a potentially decreasing reactivity towards electrophilic singlet oxygen. This qualitative scale relies on the structural analysis of the compounds 1a-f and the identification of any potential strong stereoelectronic or delocalization effects, yet a more precise scale was required.

The structures of HD, CEES and 1a-f were optimized at the $\mathrm{MP2} / 6-31+\mathrm{G}^{* *}$ level of theory in ethanol. In order to have more precise information on the availability of the nonbonding $n_{s}$ orbitals on the sulfur atom of each of these thioethers, NBO and electronic population analyses were carried out at the $B 3 L Y P / 6-31+G * *$ level of theory on the optimized structures (MP2/6-31+G**) A second order perturbation theory analysis of the Fock matrix in the NBO basis provided a convenient metric (Figure 4) to sense stereoelectronic features that are responsible for a decreased availability of the non-bonding $n_{S}$ orbitals on the sulfur atom, thus indicating a lower efficiency for the reaction with singlet oxygen. Only the potential donor/acceptor interactions $\left(E^{i n t}\right)$ involving the non-bonding $n_{S}$ and acceptors such as $\sigma^{*}{ }_{C-x}(X=$ $\mathrm{C}, \mathrm{Cl}$ ) or $\pi^{*}{ }_{\mathrm{C}=\mathrm{c}}$ were looked at on the most stable conformers of CEES, HD and 1a-f.

From the NBO and population analyses (Figure 4), it can be seen that dialkyl sulfides such as CEES, HD and 1a have welllocalized HOMO centered on the sulfur atom and are characterized by low to negligible donor/acceptor interactions (Figure 4).

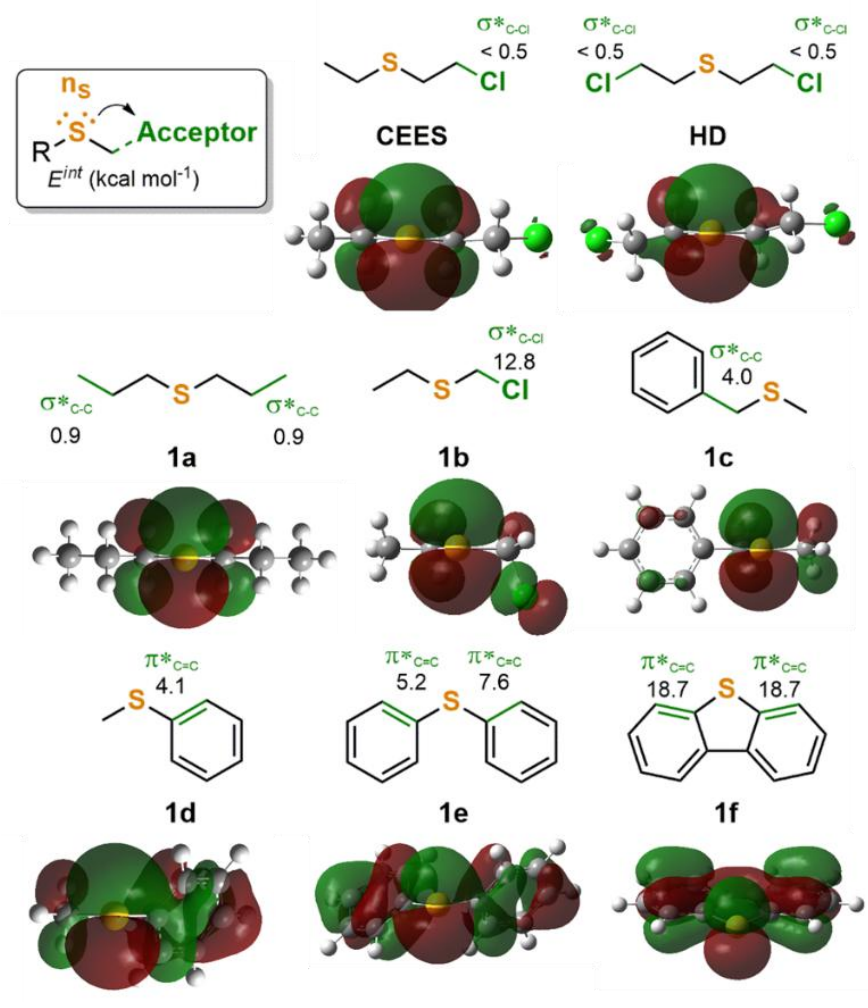

Fig. 4. Selection of model thioethers 1a-f for the computational study. The stereoelectronic interaction between a donor non-bonding $n_{\mathrm{S}}$ and a vicinal acceptor $\left(\sigma^{*}{ }_{c-X}(X=C, C l)\right.$ or $\pi^{*}{ }_{c=c}$ were looked at on the most stable conformers of CEES, HD and 1a-f) is expressed in $\mathrm{kcal} \mathrm{mol}^{-1}$. HOMO (isovalue 0.02) are represented for thioethers CEES, HD and $\mathbf{1 a - f .}$

By contrast, chloromethyl ethyl sulfide (1b) features a strongly interacting non-bonding $n_{\mathrm{s}}$ in the direct vicinity of a $\sigma^{*}{ }_{c-c l}$ acceptor and a less localized HOMO. Benzyl methyl sulfide (1c) features a medium stereoelectronic effect $\left(E^{\text {int }}=4.0 \mathrm{kcal} \mathrm{mol}^{-1}\right)$ involving a neighboring $\sigma^{*}{ }_{c-c}$ orbital. Major differences appear for $\mathbf{1 d}$ and to a larger extent to $\mathbf{1 e , f}$, where the delocalization of the non-bonding $n_{\mathrm{S}}$ orbitals to the aromatic ring drastically affects their availability. Dibenzothiophene 1 f comes with a very strong stereoelectronic interaction $\left(E^{\text {int }}=18.7 \mathrm{kcal} \mathrm{mol}^{-1}\right.$ for each $n_{\mathrm{S}}$ orbital). The results from the NBO and population analyses are in agreement with the data from the literature, which emphasize that alkyl aryl and diaryl sulfides are considered as poor quenchers of singlet oxygen compared to dialkyl sulfides due to their HOMO only partially localized on the sulfur atom. $^{67}$

The transition state for the quenching of singlet oxygen by thioethers 1a, CEES and HD towards the corresponding peroxysulfoxide intermediates A (1Aa, CEESOO and HDOO, respectively) were located at the MP2/6-31+G** level of theory in ethanol. The structure of the targeted sulfoxides ( $2 a$, CEESO and HDO) are illustrated for the sake of comparison (Figure 5). These TSs display an orthogonal approach of singlet oxygen relative to the thioether substrates and are structurally related (Supporting Information Section 4). 


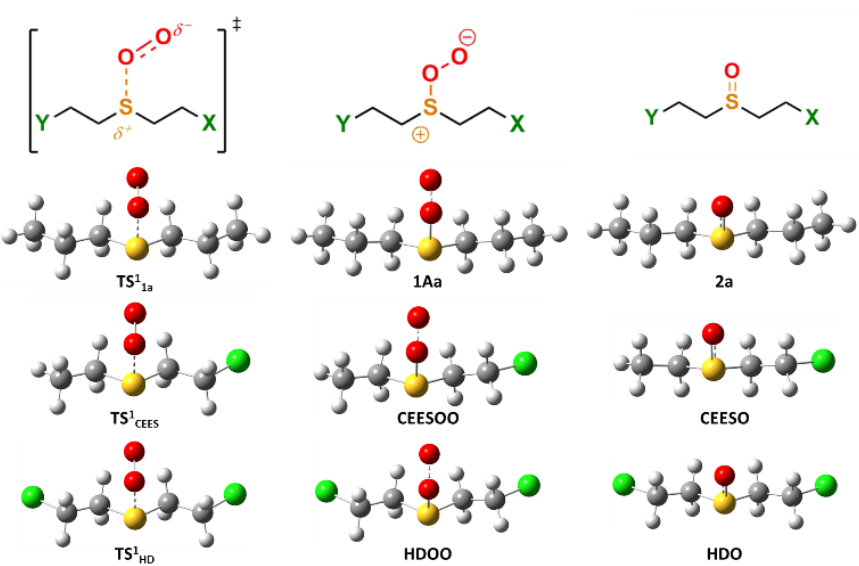

Fig. 5. Structural features of the transition states for the reaction of thioethers 1a, CEES, and HD with singlet oxygen, of the corresponding peroxysulfoxide intermediates $\mathbf{A}$ and of the final sulfoxides. See Supporting Information Section 4 for details. The corresponding $\mathrm{S}-\mathrm{O}$ and $\mathrm{O}-\mathrm{O}$ bond lengths are listed here (values in $\AA$ ): TS $^{1}{ }_{1 \mathrm{a}}$ (S-O: $\left.1.88,0-0: 1.36\right), \mathrm{TS}^{1}{ }_{\text {CEES }}$ (S-O: 1.87, O-O: 1.36), TS $^{1}{ }_{\text {HD }}$ (S-O: 1.85, O-O: 1.36), 1Aa (S-O: 1.63, O-O: 1.46), CEESOO (S-O: 1.63, O-O: 1.46), HDOO (S-O: 1.63, O-O: 1.46), 2a (S-O: 1.54), CEESO (S-O: 1.54), and HDO (S-O: 1.54).

The structural and energetic features of these TSs are in agreement with the work of Jensen and Clennan on other dialkyl thioethers. ${ }^{63-65,68,70,75-77}$ The TSs connecting 1a, CEES and $\mathrm{HD}\left(\mathrm{TS}^{1}{ }_{1 \mathrm{a}}, \quad \mathbf{T S}^{1}{ }_{\text {CEES }}\right.$ and $\mathbf{T S}^{1}{ }_{\mathrm{HD}}$, respectively) to the corresponding peroxysulfoxide intermediates (1Aa, CEESOO and HDOO) feature a rather long S-O bond (1.85-1.87 $\AA$ ) indicative of an early transition state and strong interactions between the non-bonding $n_{S}$ orbitals and singlet oxygen. ${ }^{77}$ The slightly shorter $\mathrm{S}-\mathrm{O}$ bond length for $\mathbf{T S}^{1}{ }_{\text {HD }}(1.85 \AA$ ) compared to $\mathbf{T S}^{1}{ }_{1 \mathrm{a}}, \mathbf{T S}^{1}{ }_{\text {CEES }}(1.87 \AA)$ reflects the presence of two electrons withdrawing $\mathrm{Cl}$ atoms. From the analysis of both NBO and TS structures, it appeared that both 1a and CEES are potentially good structural mimics of the parent HD for such transformation, hence the results collected on the photooxidation of both $\mathbf{1 a}$ and CEES are expected to be transposable on HD.

Based on the computational results, preliminary trials were carried out under photosensitized conditions to calibrate the

Table 2. Calibration of the photooxidation process with singlet oxygen on model thioethers (see also Figure 6 for the general setup)

\begin{tabular}{|c|c|c|c|c|}
\hline Entry & $\begin{array}{l}\text { Thioether } \\
\text { Substrate }\end{array}$ & $\begin{array}{c}\text { Solvent } \\
\text { (M) }\end{array}$ & $\begin{array}{l}\text { Catalyst } \\
\text { (mol\%) }\end{array}$ & $\begin{array}{l}\text { Conv. } \\
(\%)^{\mathrm{c}}\end{array}$ \\
\hline 1 & $1 a$ & EtOH (1) & $\mathrm{MB}(0.06)^{\mathrm{a}}$ & $>99^{d}$ \\
\hline 2 & $1 c$ & $\mathrm{EtOH}(1)$ & $\mathrm{MB}(0.06)^{\mathrm{a}}$ & $>99^{\mathrm{e}}$ \\
\hline 3 & $1 d$ & $\mathrm{EtOH}(1)$ & $\operatorname{MB}(0.06)^{a}$ & $>99^{\mathrm{e}}$ \\
\hline 4 & $1 e$ & EtOH (1) & $\mathrm{MB}(0.06)^{\mathrm{a}}$ & $5^{e}$ \\
\hline 5 & If & $\begin{array}{c}\text { EtOH:2MeTHF } \\
(0.1)\end{array}$ & $\mathrm{MB}(0.06)^{\mathrm{a}}$ & $0^{e}$ \\
\hline 6 & $1 e$ & $\mathrm{CH}_{3} \mathrm{CN}$ & $\mathrm{DCA}^{\mathrm{b}}$ & 47 \\
\hline 7 & 1f & $\mathrm{CH}_{3} \mathrm{CN}$ & $D_{C A}{ }^{b}$ & 49 \\
\hline
\end{tabular}

${ }^{\mathrm{a}} \mathrm{MB}=$ Methylene Blue, Irradiation at $610 \mathrm{~nm} ;{ }^{\mathrm{b}} \mathrm{DCA}=9,10$-dicyanoanthracene, Irradiation at $395 \mathrm{~nm} ;{ }^{\mathrm{C}}$ Conditions (see Scheme 1): $25^{\circ} \mathrm{C}, 10$ min residence time, $9 \mathrm{bar}$; the selectivity towards the corresponding sulfoxides is given in the Supporting Information (Table S3); ${ }^{d}$ Determined by GC-FID. ${ }^{e}$ Determined by HPLC-DAD. singlet oxygen oxidation process. The flow setup is described in Figure 6. It consisted of 5 specialty glass fluidic modules (FMs) of $2.6 \mathrm{~mL}$ each connected in series for a total of $13 \mathrm{~mL}$. Each glass FM features a succession of high performance static mixers to ensure high mass transfer efficiency between gaseous and liquid effluents. Each FM is integrated with a double layer heat exchanger and is sandwiched between two LED panels, each equipped with 30 high power LEDs of a defined wavelength (300 LEDs in total). The LED panels are integrated with a dedicated heat exchanger. The combination of high-efficiency static mixers and high power LEDs makes this reactor configuration particularly well suited for the generation of singlet oxygen in the presence of organic substrates. ${ }^{59}$ The reactor setup was kept under 9 bar of counterpressure with a dome-type backpressure regulator (BPR). In-line low field NMR or IR spectrometers were optionally inserted downstream for qualitative process monitoring and samples were analyzed off-line either by GC or HPLC (Supporting Information Section 2.2.1).

Solutions of representative commercial thioethers were prepared in EtOH (1 M), unless solubility issues imposed a cosolvent or a lower concentration (Supporting Information Section 2.2.3), in the presence of an organic photosensitizer (Figure 6 and Table 2). Methylene Blue (MB) was selected as organic photosensitizer for the singlet oxygen reaction with thioethers 1a-f rather than Rose Bengal for its improved resistance to photobleaching and its overall better performances as an organic photosensitizer (Supporting Information Section 3.2.3). MB is a non-toxic, stable, widely available and affordable organic photosensitizer. The liquid feed solution was mixed with oxygen upon irradiation with the appropriate wavelength at $25{ }^{\circ} \mathrm{C}$ under 9 bar of counterpressure for a total estimated residence time of $10 \mathrm{~min}$ (see Supporting Information Sections 2.2.3 and 3.1 for details). The first set of conditions involved MB (0.06 mol\%) in conjunction with oxygen and irradiation at $610 \mathrm{~nm}$ (orange LEDs) for the generation of singlet oxygen (entries 1-5). Control experiments in the absence of light or in the absence of organic photosensitizer were carried out as well, and no conversion was detected. Compound $\mathbf{1} \mathbf{b}$ was not tested since it reacted quickly with the solvent under these conditions.

The experimental observations were analyzed in parallel with the computation data (Table 2). It can be concluded that thioethers 1a, 1c and $\mathbf{1 d}$ that are characterized by a low stereoelectronic involvement of the non-bonding $n_{\mathrm{s}}$ orbitals $\left(E^{i n t} \leq 4.1 \mathrm{kcal} \mathrm{mol}^{-1}\right)$ and a rather $S$-centered HOMO are efficient quenchers of singlet oxygen under intensified flow conditions, and are converted selectively and quantitatively to the corresponding sulfoxides (Table 2, entries 1-3). No traces of sulfones or other oxidative fragmentation products were detected. By contrast, diaryl thioethers $\mathbf{1 e , f}$ were barely reactive towards singlet oxygen (entries 4,5 ). Changing the photosensitizer to 9,10-dicyanoanthracene (0.06 mol\%) in acetonitrile under irradiation at $395 \mathrm{~nm}$ (entries 6,7) led to 47 and $49 \%$ conversion for compounds $\mathbf{1 e}$,f. Such conditions are typically associated with the generation of a superoxide and an electron transfer mechanism for the oxidation of thioethers 
rather than a singlet oxygen oxidation, ${ }^{67,69,78}$ hence emphasizing the differences in terms of mechanism and its implication for the oxidation of structurally diverse thioethers. The choice of the involved mechanism depends not only on the structural and stereoelectronic features of the thioether, but also on the nature of the photosensitizer, which was not evaluated with the computational approach, hence showing the interests for combining computational with experimental data.

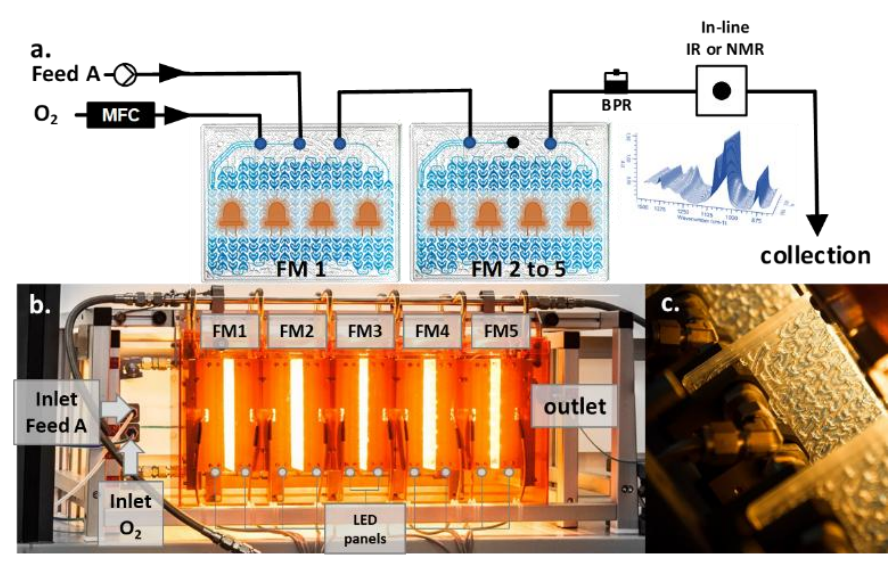

Fig. 6. a. Simplified flow chart for the photooxidation of thioethers including CEES $(B P R=$ back pressure regulator; $\mathrm{MFC}=$ mass flow controller; $\mathrm{FM}=$ fluidic module; Feed $\mathrm{A}=$ substrate typically $1 \mathrm{M}$ in EtOH with $0.06 \mathrm{~mol} \%$ of an organic photosensitizer; heat exchange on the reactive path and LED panels are omitted for clarity). b. Photograph of the low footprint and mobile chemical neutralization technology for the oxidative neutralization of mustard gas simulant CEES upon irradiation at $610 \mathrm{~nm}$ (Corning ${ }^{\circledR}$ AdvancedFlow $^{\text {TM }}$ G1/LF skid Photo Reactor; Courtesy of Corning ${ }^{\circledR}$ ). The footprint of the setup is $\mathrm{L} \times \mathrm{W} \times \mathrm{H} 94 \times 42 \times 40 \mathrm{~cm}$. c. Details of a glass fluidic module with the LED panels removed.

A stock solution of CEES (1 M) and Methylene Blue (560 $\mu \mathrm{M}$ ) weas prepared in EtOH (Supporting Information Section 2.2.3) and the process conditions were further optimized. Given the complex reactivity profile of CEES, a variety of side products are expected according to the data of the literature. Under the experimental conditions of this report, 4 impurities were detected (Scheme 1, Table 3), among which only $\mathrm{CEESO}_{2}$ (the corresponding overoxidized sulfone) is considered as toxic since it potentially produces electrophilic vinyl sulfone EVS (Figure 2). Impurities I-1 and I-3 are formed through the addition of EtOH on CEES and CEESO and can therefore be considered as neutralized species. Impurity $\mathbf{I - 2}$ is formed through the dechlorination of CEESO and is typically considered as a non-toxic chemical. The identity of all impurities was confirmed by GC, GC-MS or NMR with commercial or prepared samples.

The impact of various process parameters (irradiation time, primary source of singlet oxygen, light intensity and nature) on the efficiency and the selectivity of the photooxidation was assessed (Table 3 and Supporting Information Section 3.2). In this process, the highest conversion of CEES must be reached within a short residence time, and the oxidation must be selective enough to produce low amounts of $\mathrm{CEESO}_{2}$. The emergence of other impurities such as $\mathbf{I - 1}, \mathbf{I}-\mathbf{2}$ and $\mathbf{I}-\mathbf{3}$ does not impact negatively the process, since they are considered as neutralized species as CEESO.

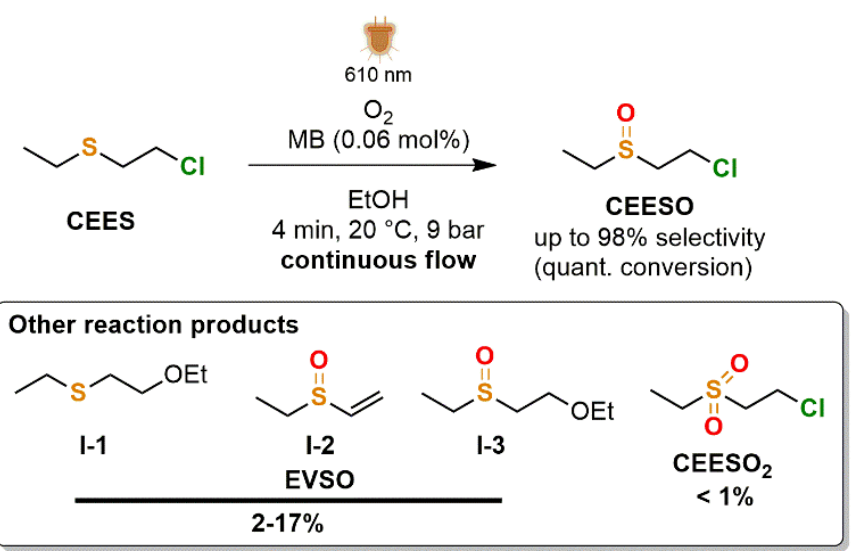

Scheme 1. Oxidative neutralization of CEES under optimized continuous flow conditions (see also Table 3).

The irradiation time can be reduced from 10 to $4 \mathrm{~min}$ upon irradiation at $610 \mathrm{~nm}$ with full intensity in the presence of oxygen while still reaching almost quantitative conversion of CEES (entries 1 and 7, >99\% and 92\%, respectively) towards mainly CEESO and other minor impurities (Scheme 1). A further decrease to $2 \mathrm{~min}$ nevertheless left about $18 \%$ of unreacted CEES in the reactor effluent ( $82 \%$ conversion). The amount of $\mathrm{CEESO}_{2}$ increased with the conversion of CEES to CEESO, yet it remained under $1 \%$ : $0.1 \%$ ( $2 \mathrm{~min}$ ), $0.4 \%$ (4 min) and $0.9 \%$ (10 $\mathrm{min})$. Impurities I-1 (3\%), I-2 (1\%) and I-3 (3\%) were present in the reactor effluent from the experiment at 4 min of irradiation time. 
Table 3. Optimization of the oxidative neutralization of CEES under continuous flow conditions (see also Scheme 1).

\begin{tabular}{|c|c|c|c|c|c|c|c|c|c|c|}
\hline \multirow[t]{2}{*}{ Entry } & \multirow[t]{2}{*}{ Oxidant } & \multirow{2}{*}{$\begin{array}{c}\text { RT } \\
(\mathrm{min})\end{array}$} & \multirow{2}{*}{$\begin{array}{l}\text { LEDs } \\
(\mathrm{nm})\end{array}$} & \multirow{2}{*}{$\begin{array}{c}\text { Conv. }^{c} \\
(\%)\end{array}$} & \multicolumn{6}{|c|}{ Reactor effluent composition (\%) ${ }^{d}$} \\
\hline & & & & & CEES & CEESO & $\mathrm{CEESO}_{2}$ & $\mathrm{I}-1$ & $1-2$ & $1-3$ \\
\hline 1 & $\mathrm{O}_{2}$ & 10 & 610 & $>99^{a}$ & 0 & 95 & 1 & 0 & 1 & 3 \\
\hline 2 & $\mathrm{O}_{2}$ & 10 & white & $>99^{a}$ & 0 & 96 & 1 & 0 & 2 & 1 \\
\hline 3 & $\mathrm{O}_{2}$ & 10 & white & $>99^{a}$ & 0 & 95 & 1 & 0 & 1 & 3 \\
\hline 4 & Air & 10 & 610 & $>99^{a}$ & 0 & 84 & 1 & 0 & 1 & 15 \\
\hline 5 & Air & 10 & 610 & $>99^{b}$ & 0 & 85 & 0 & 0 & 1 & 14 \\
\hline 6 & $\mathrm{O}_{2}$ & 4 & 610 & $99^{a}$ & 1 & 92 & 0 & 3 & 1 & 3 \\
\hline 7 & Air & 4 & 610 & $51^{a}$ & 81 & 14 & 0 & 4 & 0 & 0 \\
\hline 8 & $\mathrm{O}_{2}$ & 4 & white & $>99^{a}$ & 0 & 98 & 1 & 0 & 2 & 0 \\
\hline 9 & Air & 4 & white & $68^{a}$ & 41 & 55 & 0 & 3 & 1 & 0 \\
\hline 10 & $\mathrm{O}_{2}$ & 2 & 610 & $82^{a}$ & 28 & 68 & 0 & 2 & 1 & 0 \\
\hline
\end{tabular}

${ }^{\mathrm{a}} 1 \mathrm{M}$ solution in $\mathrm{EtOH}$ with $0.06 \mathrm{~mol} \%$ Methylene Blue (Rose Bengal for entry 3), processed at $20^{\circ} \mathrm{C}$ under 9 bar of counterpressure; ${ }^{\mathrm{b}} 0.1 \mathrm{M}$ solution in EtOH with $0.06 \mathrm{~mol} \%$ Methylene Blue, processed at $20^{\circ} \mathrm{C}$ under 9 bar of counterpressure; ${ }^{\mathrm{C}}$ Determined by GC with an external calibration on commercial CEES; ${ }^{\mathrm{d}}$ Ratio of compounds determined by GC. The references for compounds CEESO, CEESO, I-1, I-2 and I-3 were either purchased from commercial sources or synthesized in the lab.

At 10 min of irradiation time, only impurities I-2 (1\%) and I$3(3 \%)$ were detected besides $\mathbf{C E E S O}_{2}$. It is expected that the longer irradiation time oxidized completely $\mathbf{~} \mathbf{- 1}$ towards I-3. At the shortest residence time $(2 \mathrm{~min})$, impurity $\mathbf{I - 3}$ was not detected while impurities $\mathbf{I}-\mathbf{1}$ and $\mathbf{I}-\mathbf{2}$ reached $2 \%$ and $1 \%$, respectively. These observations indicate a potential filiation between I-3 and I-1, as well as I-2 (EVSO) and CEESO, although other reaction paths might be incriminated (See Supporting Information Section 2.2.4). Impurity I-2 is also believed to arise from the specific reactivity of the corresponding hydroperoxysulfonium ylide $\mathbf{B}_{\mathbf{2}}$ intermediate (Figure 3$)^{80}$ Impurity I-1 most likely arises from a slow reaction between the substrate CEES and the solvent upon storage of the feed solution. Such reaction was not significant upon standing in the feed, and is likely to be accelerated in the flow reactor with the local heat generated by the LEDs (Supporting Information Section 2.2.4).

The efficiency of the photooxidation is typically greater with pure oxygen rather than air. This is logical and explainable as the proportion of oxygen is simply lower in air. Within $4 \mathrm{~min}$ of irradiation at $610 \mathrm{~nm}$ (full intensity), the conversion of CEES reached $99 \%$ with pure oxygen while it decreased to $51 \%$ with air under the same conditions. For the longest irradiation time (10 $\mathrm{min}$ ), either oxygen or air gave a similar conversion (99\%). The reactor effluent from the experiment with air within $4 \mathrm{~min}$ of irradiation was exempt from $\mathrm{CEESO}_{2}$, although this is most likely related to the low conversion of CEES than to the nature of the primary source of singlet oxygen. The amount of impurity 1-1, was slightly higher for the experiment with air (4\%) than for the experiment with oxygen (3\%). Impurity I-3 was absent from the reaction with air, while $3 \%$ were formed in the presence of oxygen.

The intensity and the nature of the light source were also looked at (Supporting Information Section 3.2.4). Decreasing the LEDs intensity typically led to lower conversions, and the maximum intensity was used for most of the trials. With oxygen or air at the longest irradiation time (10 $\mathrm{min})$, either orange LEDs (610 $\mathrm{nm}$ ) or white LEDs (4000K) provided $>99.9 \%$ conversion of CEES. The reactor effluent from the experiment with the $610 \mathrm{~nm}$ LEDs contained 95\% of CEESO, and traces of $\mathrm{CEESO}_{2}(0.6 \%), \mathrm{I}-1$ (0.5\%), I-2 (2\%) and I-3 (1\%). The reactor effluent from the experiment with white LEDs had a similar composition with $96 \%$ of CEESO, and traces of CEESO 2 (1\%), I-2 (1\%) and I-3 (3\%). Decreasing the residence time to $4 \mathrm{~min}$ still afforded quantitative conversion of CEES under white LEDs irradiation. In the latter case, the crude reactor effluent contained $98 \%$ of CEESO along with $0.5 \%$ of $\mathrm{CEESO}_{2}$ and less than $2 \%$ of $\mathbf{1 - 2}$. Pure oxygen could be replaced with air, although the concentration of the solution had to be decreased 10 -fold to maintain full conversion within $10 \mathrm{~min}$ of residence time. Within $4 \mathrm{~min}$ of irradiation in the presence of air, white LEDs gave better photooxidation performances than the orange LEDs, affording $68 \%$ conversion in CEES $(51 \%$ under irradiation at $610 \mathrm{~nm}$ ). Both samples were free of $\mathrm{CEESO}_{2}$.

To summarize the optimization of the chemical oxidative neutralization of CEES, the best results involved process conditions relying on oxygen under $4 \mathrm{~min}$ irradiation either at $610 \mathrm{~nm}$ or under white light at room temperature and 9 bar of counterpressure. Under these conditions, only a trace amount of $\mathrm{CEESO}_{2}$ is formed. Air can also be utilized for the neutralization of CEES, affording complete conversion of CEES yet with a longer irradiation time (10 $\mathrm{min})$.

\section{Conclusion}

This work illustrates the development of a low footprint, mobile, robust and frugal chemical neutralization technology for the oxidative neutralization of a mustard gas simulant. It relies on the unique features of a highly engineered continuous flow setup and robust conditions using non-toxic and widely available chemicals. The experimental work is also supported with a computational rationalization of the reactivity of selected thioethers towards singlet oxygen. The optimized conditions use concentrated solutions of CEES in 
ethanol with a trace amount of Methylene Blue upon irradiation with orange or white light. Quantitative conversion was obtained within $4 \mathrm{~min}$ of irradiation time under mild process conditions $\left(20^{\circ} \mathrm{C}, 9 \mathrm{bar}\right)$. Oxygen or air can be utilized as primary oxidant, although with air longer irradiation times or lower feed concentrations are typically required. Under optimized conditions, only trace amounts of toxic $\mathrm{CEESO}_{2}$ are detected in the reactor effluent, hence providing an extremely cost effective, operator-friendly and low footprint process for the oxidative neutralization of CEES. The neutralization technology can be embarked on a vehicle for on-site interventions, localized at a neutralization facility or both. The ability to transpose these optimized conditions to larger scales with commercial Corning Advanced-Flow Reactors with minimal reoptimization definitively opens up new perspectives for the safe chemical neutralization of mustard gas with a minimal footprint under frugal conditions. Such a protocol of neutralization could possibly be further adapted to other $S$ based CWA from the V-series (VX, VR).

\section{Conflicts of interest}

There are no conflicts to declare.

\section{Acknowledgments}

This work was supported by the University of Liège (Welcome Grant WG-13/03, JCMM) and by the French Community of Belgium (Concerted Research Action ARC-icFlow, Integrated Continuous Flow Photoreactors). Computational resources have been provided by the "Consortium des Équipements de Calcul Intensif" (CÉCl), funded by the "Fonds de la Recherche Scientifique de Belgique" (F.R.S.-FNRS) under Grant No. 2.5020.11. Dr. Morgan Hans is acknowledged for carrying out the $\mathrm{GC} / \mathrm{MS}$ experiments.

\section{Notes and references}

1 https://www.iss.europa.eu/content/preventing-reemergence-chemicalweapons\#_a_short_history_of_chemical_warfare.

2 B. Friedrich, D. Hoffmann, J. Renn, F. Schmaltz and M. Wolf, One hundred years of chemical warfare: Research, deployment, consequences, 2017.

3 https://www.opcw.org/chemical-weaponsconvention/download-convention.

4 https://www.opcw.org/about-us.

5 R. Gérardy, N. Emmanuel, T. Toupy, V. E. Kassin, N. N. Tshibalonza, M. Schmitz and J. C. M. Monbaliu, European J. Org. Chem., 2018, 2018, 2301-2351.

6 https://www.armscontrol.org/factsheets/cbwprolif.

7 https://www.opcw.org/media-centre/opcw-numbers.

8 https://www.opcw.org/chemical-weaponsconvention/annexes/annex-chemicals/schedule-1. Q. Q. Wang, R. A. Begum, V. W. Day and K. Bowman-James, Org. Biomol. Chem., 2012, 10, 8786-8793.
Decontamination of Warfare Agents, 2008.

B. Picard, I. Chataigner, J. Maddaluno and J. Legros, Org. Biomol. Chem., 2019, 17, 6528-6537.

https://www.opcw.org/our-work/eliminating-chemicalweapons.

N. Karton-Lifshin, S. Katalan, I. Columbus, R. Chen, L. Yehezkel, M. Madmon, S. Dagan, S. Elias, G. Fridkin and Y. Zafrani, Chem. Commun., 2019, 55, 12471-12474.

G. W. Wagner, L. R. Procell, Y. C. Yang and C. A. Bunton, Langmuir, 2001, 17, 4809-4811.

S. R. Livingston and C. C. Landry, J. Am. Chem. Soc., 2008, 130, 13214-13215.

F. Carniato, C. Bisio, R. Psaro, L. Marchese and M. Guidotti, Angew. Chemie - Int. Ed., 2014, 53, 10095-10098.

Y. Hou, H. An, Y. Zhang, T. Hu, W. Yang and S. Chang, ACS Catal., 2018, 8, 6062-6069.

X. Li, J. Dong, H. Liu, X. Sun, Y. Chi and C. Hu, J. Hazard. Mater., 2018, 344, 994-999.

J. Dong, J. Hu, Y. Chi, Z. Lin, B. Zou, S. Yang, C. L. Hill and C. Hu, Angew. Chemie - Int. Ed., 2017, 56, 4473-4477.

B. Picard, B. Gouilleux, T. Lebleu, J. Maddaluno, I. Chataigner, M. Penhoat, F. X. Felpin, P. Giraudeau and J. Legros, Angew. Chemie - Int. Ed., 2017, 56, 7568-7572. R. B. and F. E. Sophie Mohamed, Sebastien Balieu, Emilie
Petit, Ludovic Galas, Damien Schapman, Julie Hardouin,
ChemCom, 2019, 55, 13243-13246.

Z. Wang, H. Xi, L. Kong, Y. Zuo, Z. Shi and S. Zhao, Mol. Catal., 2017, 430, 1-8.

Y. Liu, A. J. Howarth, J. T. Hupp and O. K. Farha, Angew. Chemie - Int. Ed., 2015, 54, 9001-9005.

W. Q. Zhang, K. Cheng, H. Zhang, Q. Y. Li, Z. Ma, Z. Wang, J. Sheng, Y. Li, X. Zhao and X. J. Wang, Inorg. Chem., 2018, 57, 4230-4233.

L. Ma, Y. Liu, Y. Liu, S. Jiang, P. Li, Y. Hao, P. Shao, A. Yin, X. Feng and B. Wang, Angew. Chemie - Int. Ed., 2019, 58, 4221-4226.

J. R. Hiscock, G. P. Bustone and E. R. Clark, ChemistryOpen, 2017, 6, 497-500.

H. K. Dong, M. Kim and S. Chang, Org. Lett., 2005, 7, 50145018.

Y. Liu, C. T. Buru, A. J. Howarth, J. J. Mahle, J. H. Buchanan, J. B. Decoste, J. T. Hupp and O. K. Farha, J. Mater. Chem. A, 2016, 4, 13809-13813.

C. R. Ringenbach, S. R. Livingston, D. Kumar and C. C. Landry, Chem. Mater., 2005, 17, 5580-5586.

R. T. Gephart, P. N. Coneski and J. H. Wynne, ACS Appl. Mater. Interfaces, 2013, 5, 10191-10200.

J. T. Rhule, W. A. Neiwert, K. I. Hardcastle, B. T. Do and C. L. Hill, J. Am. Chem. Soc., 2001, 123, 12101-12102.

B. Smolkin, N. Levi, N. Karton-Lifshin, L. Yehezkel, Y. Zafrani and I. Columbus, J. Org. Chem., 2018, 83, 13949-13955.

C. T. Buru, M. B. Majewski, A. J. Howarth, R. H. Lavroff, C. W. Kung, A. W. Peters, S. Goswami and O. K. Farha, ACS Appl. Mater. Interfaces, 2018, 10, 23802-23806.

A. J. Howarth, C. T. Buru, Y. Liu, A. M. Ploskonka, K. J. Hartlieb, M. McEntee, J. J. Mahle, J. H. Buchanan, E. M. Durke, S. S. Al-Juaid, J. F. Stoddart, J. B. DeCoste, J. T. Hupp 
and O. K. Farha, Chem. - A Eur. J., 2017, 23, 214-218.

C. F. Pereira, Y. Liu, A. Howarth, F. Figueira, J. Rocha, J. T. Hupp, O. K. Farha, J. P. C. Tomé and F. A. Almeida Paz, ACS Appl. Nano Mater., 2019, 2, 465-469. K. Ma, T. Islamoglu, Z. Chen, P. Li, M. C. Wasson, Y. Chen, Y. Wang, G. W. Peterson, J. H. Xin and O. K. Farha, J. Am. Chem. Soc., 2019, 141, 15626-15633. J. Dad'ová, E. Svobodová, M. Sikorski, B. König and R. Cibulka, ChemCatChem, 2012, 4, 620-623. T. Neveselý, E. Svobodová, J. Chudoba, M. Sikorski and R. Cibulka, Adv. Synth. Catal., 2016, 358, 1654-1663. H. Wang, G. W. Wagner, A. X. Lu, D. L. Nguyen, J. H. Buchanan, P. M. McNutt and C. J. Karwacki, ACS Appl. Mater. Interfaces, 2018, 10, 18771-18777. T. S. A. Heugebaert, C. V. Stevens and C. O. Kappe, ChemSusChem, 2015, 8, 1648-1651. C. A. Hone and C. O. Kappe, The Use of Molecular Oxygen for Liquid Phase Aerobic Oxidations in Continuous Flow, Springer International Publishing, 2019, vol. 377. V. E. H. Kassin, R. Gérardy, T. Toupy, Di. Collin, E. Salvadeo, F. Toussaint, K. Van Hecke and J. C. M. Monbaliu, Green Chem., 2019, 21, 2952-2966. E. S. and J.-C. M. M. V.-E. H. Kassin, T. Toupy, G. Petit, P. Bianchi, J. Flow Chem., 2019, Accepted. D. Cambié, J. Dobbelaar, P. Riente, J. Vanderspikken, C. Shen, P. H. Seeberger, K. Gilmore, M. G. Debije and T. Noël, Angew. Chemie - Int. Ed., 2019, 58, 14374-14378. H. P. L. Gemoets, Y. Su, M. Shang, V. Hessel, R. Luque and T. Noël, Chem. Soc. Rev., 2016, 45, 83-117. T. Noël, Chem. Rev., 2016, 116, 10276-10341.

L. Suhadolnik, A. Pohar, U. Novak, B. Likozar, A. Mihelič and M. Čeh, J. Ind. Eng. Chem., 2019, 72, 178-188.

M. Krivec, A. Pohar, B. Likozar and G. Drazic, AlChE J., 2015, 61, 572-581.

L. Suhadolnik, A. Pohar, B. Likozar and M. Čeh, Chem. Eng. J., 2016, 303, 292-301.

D. Rackl, P. Kreitmeier and O. Reiser, Green Chem., 2015, 18, 214-219.

D. C. Fabry, Y. A. Ho, R. Zapf, W. Tremel, M. Panthöfer, M Rueping and T. H. Rehm, Green Chem., 2017, 19, $1911-$ 1918.

P. R. Adiyala, S. Jang, N. K. Vishwakarma, Y.-H. Hwang and D.-P. Kim, Green Chem., , DOI:10.1039/c9gc03496j.

A. Steiner, J. D. Williams, O. De Frutos, J. A. Rincón, C. Mateos and C. O. Kappe, Green Chem., 2020, 22, 448-454. M. B. Plutschack, B. Pieber, K. Gilmore and P. H. Seeberger, Chem. Rev., 2017, 117, 11796-11893.

H. Ishitani, Y. Saito, B. Laroche, X. Rao and S. Kobayashi, Chapter 1: Recent Perspectives in Catalysis under Continuous Flow, 2020, vol. 2020- January.

M. Berton, J. M. de Souza, I. Abdiaj, D. T. McQuade and D. R. Snead, J. Flow Chem., 2020, 10, 73-92.

J. J. Kilbane and K. Jackowski, J. Chem. Technol. Biotechnol., 1996, 65, 370-374.

F. Lévesque and P. H. Seeberger, Org. Lett., 2011, 13, 5008-5011.
N. Emmanuel, C. Mendoza, M. Winter, C. R. Horn, A. Vizza, L. Dreesen, B. Heinrichs and J. C. M. Monbaliu, Org. Process Res. Dev., 2017, 21, 1435-1438.

C. Mendoza, N. Emmanuel, C. A. Páez, L. Dreesen, J. C. M. Monbaliu and B. Heinrichs, J. Photochem. Photobiol. A Chem., 2018, 356, 193-200.

C. Mendoza, N. Emmanuel, C. A. Páez, L. Dreesen, J.-C. M. Monbaliu and B. Heinrichs, ChemPhotoChem, 2018, 2, 890897.

J. C. and D. J. F. M. J. Frisch, G. W. Trucks, H. B. Schlegel, G. E. Scuseria, M. A. Robb, J. R. Cheeseman, G. Scalmani, V. Barone, B. Mennucci, G. A. Petersson, H. Nakatsuji, M. Caricato, X. Li, H. P. Hratchian, A. F. Izmaylov, J. Bloino, G. Zheng, J. L. Sonnenberg, M. Had, Gaussian Inc., Wallingford CT.

F. Jensen, J. Org. Chem., 1992, 57, 6478-6487.

A. Greer, F. Jensen and E. L. Clenna, J. Org. Chem., 1996,

61, 4107-4110.

A. Toutchkine, D. Aebisher and E. L. Clennan, J. Am. Chem. Soc., 2001, 123, 4966-4973.

E. Baciocchi, T. Del Giacco, F. Elisei, M. F. Gerini, M. Guerra, A. Lapi and P. Liberali, J. Am. Chem. Soc., 2003, 125, 16444-16454.

S. M. Bonesi, M. Fagnoni and A. Albini, J. Org. Chem., 2004, 69, 928-935.

E. L. Clennan, S. E. Hightower and A. Greer, J. Am. Chem. Soc., 2005, 127, 11819-11826.

S. M. Bonesi, S. Crespi, D. Merli, I. Manet and A. Albini, J. Org. Chem., 2017, 82, 9054-9065.

E. L. Clennan and A. Greer, J. Org. Chem., 1996, 61, 47934797.

K. Ishiguro, M. Hayashi and Y. Sawaki, J. Am. Chem. Soc., 1996, 118, 7265-7271.

W. S. Jenks, N. Matsunaga and M. Gordon, J. Org. Chem., 1996, 61, 1275-1282.

F. Jensen, A. Greer and E. L. Clennan, J. Am. Chem. Soc., 1998, 120, 4439-4449.

S. M. Bonesi, M. Mella, N. D'Alessandro, G. G. Aloisi, M. Vanossi and A. Albinit, J. Org. Chem., 1998, 63, 9946-9955. . Clennan, J. Am. Chem. Soc, 1999, 121, 2915-2916.

A. Toutchkine and E. L. Clennan, J. Am. Chem. Soc., 2000, 122, 1834-1835.

E. L. Clennan, Acc. Chem. Res., 2001, 34, 875-884.

S. M. Bonesi, I. Manet, M. Freccero, M. Fagnoni and A. Albini, Chem. - A Eur. J., 2006, 12, 4844-4857. C. G. and C. S. Foote, J. Am. Chem. Soc., 1982, 104, 60606063. 


\section{Table of Content}

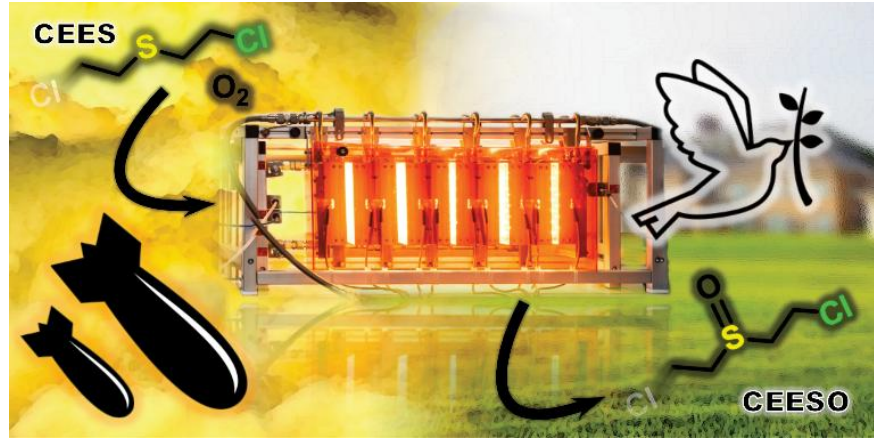

Intensified chemical neutralization of chemical warfare agent simulant CEES with air and light under continuous flow conditions 